\title{
Protein-losing Enteropathy Caused by Intestinal or Colonic Lymphangiectasia Complicated by Sporadic Lymphangioleiomyomatosis: A Report of Two Cases
}

\author{
Koichi Nishino ${ }^{1,4}$, Kaku Yoshimi ${ }^{1,4}$, Tomoyoshi Shibuya ${ }^{2}$, Takuo Hayashi ${ }^{3,4}$, Keiko Mitani ${ }^{3,4}$, \\ Etsuko Kobayashi ${ }^{1,4}$, Masako Ichikawa ${ }^{1}$, Tetsuhiko Asao ${ }^{1}$, Yohei Suzuki ${ }^{1}$, Tadashi Sato ${ }^{1}$, \\ Satomi Shiota ${ }^{1}$, Yuzo Kodama ${ }^{1}$, Kazuhisa Takahashi ${ }^{1}$ and Kuniaki Seyama ${ }^{1,4}$
}

\begin{abstract}
This report describes two patients with sporadic lymphangioleiomyomatosis complicated by protein-losing enteropathy (PLE). Imaging studies indicated retroperitoneal lymphangioleiomyomas and abnormalities of the adjacent digestive tract. Endoscopic mucosal biopsy revealed colonic lymphangiectasia in one patient; whereas the site in the other patient was intestinal. Treatment with sirolimus led to the complete resolution of PLE within several months; additionally, marked shrinkage was observed in the lymphangioleiomyomas of both cases. These findings suggest that colonic or intestinal lymphatic congestion due to neighboring lymphangioleiomyomas was the mechanism for the development of PLE. At the time of writing this report, the beneficial effect of sirolimus has lasted for more than 3 years.
\end{abstract}

Key words: lymphangioleiomyomatosis, protein-losing enteropathy, intestinal lymphangiectasia, colonic lymphangiectasia, sirolimus

(Intern Med 56: 943-948, 2017)

(DOI: 10.2169/internalmedicine.56.7769)

\section{Introduction}

Lymphangioleiomyomatosis (LAM) is a rare systemic disease that almost exclusively affects young women. It is characterized by the proliferation of abnormal smooth musclelike cells (LAM cells) in the lungs and along the axial lymphatics. Chyle leakage is a common complication of LAM. Chylous pleural effusion or peritoneal effusion occurs in 10$15 \%$ of LAM patients. Furthermore, chyluria (1) and chylous vaginal discharge (2) have been reported. When chyle leaks into the gastrointestinal lumen, protein-losing enteropathy (PLE) can develop.

PLE involves an excessive loss of serum proteins into the gastrointestinal lumen, which leads to diarrhea, edema, pleural effusion, and ascites as well as the laboratory findings of reduced concentrations of albumin, gamma-globulins ( $\operatorname{IgA}$, IgG, IgM, etc.), fibrinogen, transferrin, and ceruloplasmin. Intestinal lymphangiectasia, a major cause of PLE, manifests as the dilation and rupture of intestinal lymphatic vessels, causing a loss of lymphatic fluid into the lumen of the small intestine.

Although it is extremely rare, cases of PLE complicated by LAM have been reported $(3,4)$. According to these reports, the etiology of PLE seems to be secondary intestinal lymphangiectasia via obstruction of the lymphatic ducts by retroperitoneal lymphangioleiomyomas; however, the exact mechanism underlying its development is unknown. Additionally, the long-term clinical course has not been described. We herein report two patients with LAM complicated by PLE, which was caused by secondary intestinal and colonic lymphangiectasia. Both were successfully treated

\footnotetext{
${ }^{1}$ Division of Respiratory Medicine, Juntendo University Faculty of Medicine and Graduate School of Medicine, Japan, ${ }^{2}$ Division of Gastroenterology, Juntendo University Faculty of Medicine and Graduate School of Medicine, Japan, ${ }^{3}$ Division of Human Pathology, Juntendo University Faculty of Medicine and Graduate School of Medicine, Japan and ${ }^{4}$ The Study Group of Pneumothorax and Cystic Lung Diseases, Japan Received for publication May 30, 2016; Accepted for publication August 24, 2016 Correspondence to Dr. Kuniaki Seyama, kseyama@juntendo.ac.jp
} 
with sirolimus in addition to conventional therapy.

\section{Case Reports}

\section{Case 1}

Patient 1 was a 39-year-old woman with sporadic LAM whose accompanying thoracic endometriosis had been treated with danazol for 10 months at our hospital. She subsequently complained of excreting a milky-colored liquid stool. The stool excretion was exacerbated by exercise and the intake of fatty food. Neither abdominal pain nor nausea was present. Her medical history included repeated episodes of right pneumothorax during menstruation, which had occurred since she was 26 years old. After undergoing videoassisted thoracoscopic surgery (VATS) at 30 years of age, she was diagnosed with thoracic endometriosis. Pneumothorax did not recur for 7 years after the initiation of norethisterone/mestranol, which was administered to treat her endometriosis. However, at 38 years of age, right pneumothorax occurred. Chest computed tomography (CT) showed thin-walled cysts scattered throughout the lungs (Fig. 1A); however, her pulmonary function was well-preserved (forced expiratory volume $\left(\mathrm{FEV}_{1}\right) 103.9 \%$ of the predicted, $\mathrm{FEV}_{1} /$ forced vital capacity (FVC) 91.7\%). Although the patient was diagnosed with LAM via VATS on that occasion, no findings of thoracic endometriosis were found. The aforementioned danazol treatment (instead of norethisterone/ mestranol) was initiated for endometriosis with the expectation that it may show marginal effectiveness for LAM; however, after 10 months of danazol treatment, she began excreting a milky fluid in her stool.

On physical examination, her vital signs were within the normal limits, her bilateral lung sounds were clear, the abdominal wall was soft and flat, and no pretibial pitting edema was found. No superficial lymph nodes were palpable. The laboratory data revealed lymphocytopenia (white

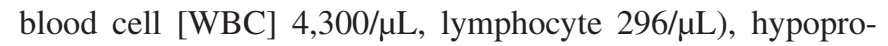
teinemia (total protein [TP] $4.0 \mathrm{~g} / \mathrm{dL}$ ), hypoalbuminemia (albumin [Alb] $2.0 \mathrm{~g} / \mathrm{dL}$ ). The data regarding the patient's renal and liver functions, and her serum immunoglobulin $G$ (IgG) level were within the normal limits. The patient's stool was a milky-colored liquid with some soft or loose materials (Fig. 1B). A biochemical analysis of the stool revealed triglyceride (TG) $944 \mathrm{mg} / \mathrm{dL}$, total cholesterol (TChol) $61 \mathrm{mg} / \mathrm{dL}$, and $\alpha_{1}$-antitrypsin (AAT) $134 \mathrm{mg} / \mathrm{dL}$, indicating that it was chylous. T2-weighted magnetic resonance imaging (MRI) of the pelvis depicted large retroperitoneal lymphangioleiomyomas and abnormalities of the sigmoid colon, including wall thickening and fluid collection (Fig. 1C). Lymphatic scintigraphy showed an accumulation of radioactive substances in the pelvic lymphangioleiomyomas and their leakage into the sigmoid colon at 120 minutes after the subcutaneous injection of ${ }^{99 \mathrm{~m}} \mathrm{Tc}-$ labeled human serum albumin (HSA) into the bilateral pedal skin (Fig. 1D). Fiberoptic colonoscopy revealed an edematous appearance of the rectal mucosa (Fig. 1E). A pathological examination of the biopsied rectal mucosa revealed dilated lymphatic vessels in the lamina propria (Fig. 1F, G).

According to these results, Patient 1 was diagnosed as having PLE caused by secondary lymphangiectasia with LAM, resulting in chylous stool, lymphocytopenia, hypoproteinemia and hypoalbuminemia. A fat-restricted diet and the subcutaneous injection of a gonadotropin-releasing hormone analogue $(\mathrm{GnRH})$, leuprorelin acetate $(1.88 \mathrm{mg}$, every four weeks), was initiated instead of danazol. However, her chylous stool remained at 6 months after the initiation of GnRH therapy. Accordingly, we decided to start the off-label use of sirolimus $(1 \mathrm{mg} /$ day; the trough level at 1 month was 1.8 $\mathrm{ng} / \mathrm{mL}$ ). The effect of sirolimus was remarkable; the chylous stool disappeared within 3 months, and her lymphocytopenia, hypoproteinemia, and hypoalbuminemia completely resolved within 6 months. The fat-restricted diet and GnRH therapy were stopped at 6 months after the initiation of sirolimus. Pelvic (T2-weighted) MRI at 2 years after the initiation of treatment showed the marked shrinkage of the pelvic lymphangioleiomyomas, while sigmoid colon appeared normal; the previous interior fluid collection had disappeared (Fig. 1H). At the time of writing this report (approximately $31 / 2$ years after the initiation of sirolimus treatment), sirolimus ( $1 \mathrm{mg} /$ day) remains effective. Regarding the adverse events of sirolimus treatment, the patient experienced oral mucositis (grade 1) transiently during the first several months of sirolimus use.

\section{Case 2}

Patient 2 was a 29-year-old woman who presented with bilateral leg edema. Huge intra-abdominal cystic tumors accompanying hypoproteinemia (TP $4.5 \mathrm{~g} / \mathrm{dL}$ ) and hypoalbuminemia (Alb $2.9 \mathrm{~g} / \mathrm{dL}$ ) were noted. She underwent surgical resection of the tumors after a diagnosis of suspected pseudomyxoma peritonei, which eventually resulted in the diagnosis of LAM. Her hypoproteinemia and leg edema were transiently ameliorated (TP $7.1 \mathrm{~g} / \mathrm{dL}$ and Alb $4.3 \mathrm{~g} / \mathrm{dL}$ ) after the operation. Two months later, she was referred to our hospital for the management of LAM. At that point, she was followed up with observation because the cystic lesions of her lung were mild.

However, hypoproteinemia and hypoalbuminemia reappeared together with leg edema at one year (TP 3.5-4.5 g/dL and Alb 1.9-3.0 g/dL), along with lymphocytopenia (190$306 / \mu \mathrm{L})$ and a decreased $\operatorname{IgG}$ level (110-199 mg/dL) in serum. Her liver function and the results of a urinalysis were normal. The use of diuretics and manual lymphatic drainage were attempted, however, the effects were marginal. At 32 years of age, she was admitted to our hospital for further examination. The stool appearance was normal, but a biochemical analysis revealed an elevated level of AAT (217 $\mathrm{mg} / \mathrm{dL}$ ). Chest CT showed a small number of thin-walled cysts in both lung fields (Fig. 2A). Abdominal MRI (T2weighted imaging) revealed multiple cystic lesions around the upper pole of the right kidney, duodenal wall thickening, 

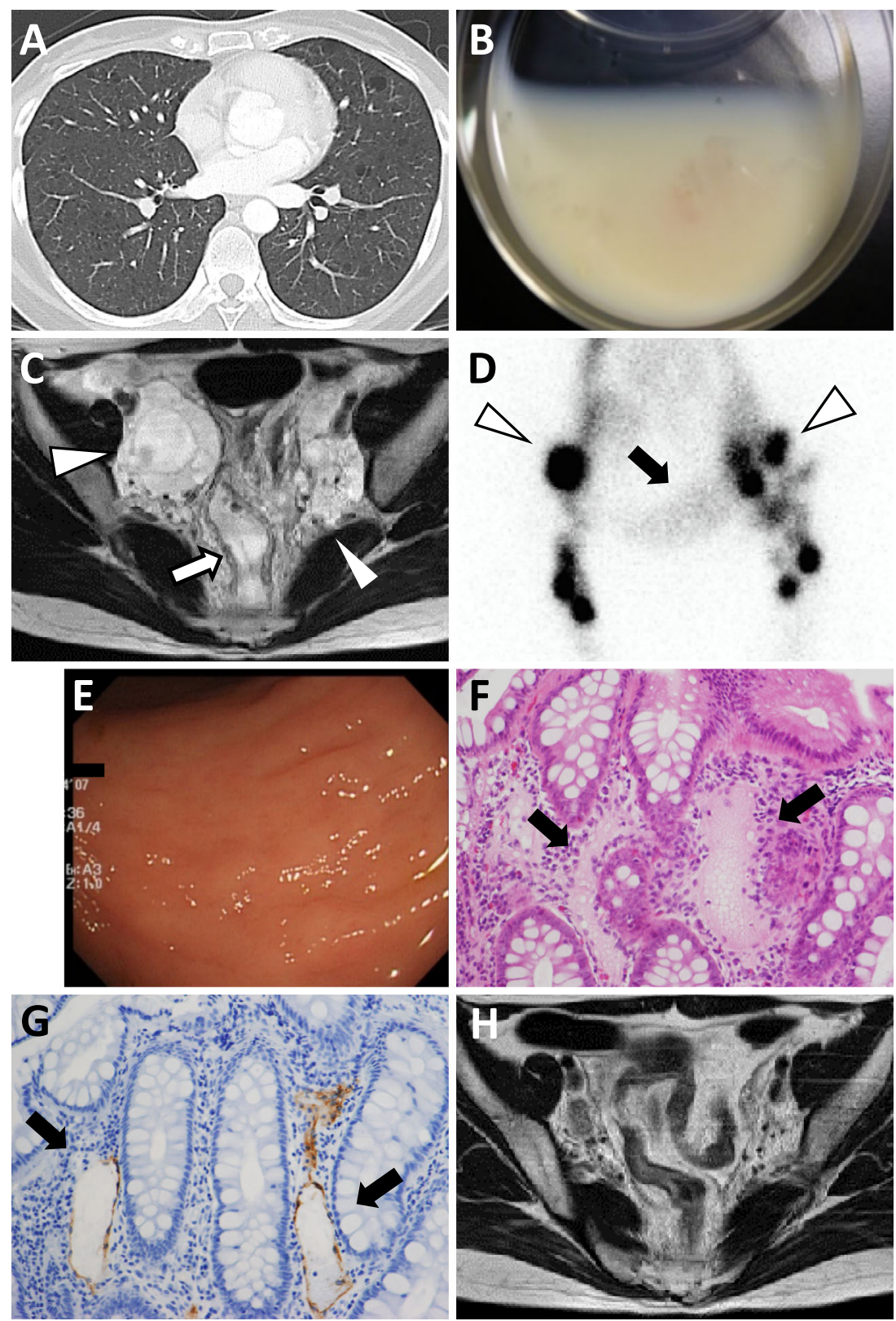

Figure 1. The clinical features of Patient 1. A: Chest CT showing thin-walled cysts scattered throughout the lungs. B: A photograph of the milky-colored liquid excreted as stool. Note the presence of soft, loose materials. C: Magnetic resonance imaging (MRI) of the pelvis revealing large cystic lymphangioleiomyomas (arrowhead) and abnormalities of the sigmoid colon, i.e., thickening of its wall and interior fluid collection (arrow) (T2-weighted imaging). D: Lymphatic scintigraphy of the accumulated radioactivity in the pelvic lymphangioleiomyomas (arrowheads) and the leakage into the sigmoid colon (arrow) at 120 minutes after the subcutaneous injection of ${ }^{99}$ Tc-labeled HSA into the bilateral pedal skin. E: Fiberoptic colonoscopy of the edematous rectal mucosa. F: The biopsied rectal mucosa with dilated, thin-walled spaces (arrows) in the lamina propria (Hematoxylin and Eosin staining, $\times$ 82) G: Anti-podoplanin antibody (clone D2-40) staining confirmed that the dilated, irregularlyshaped, thin-walled spaces were lymphatic vessels lined with lymphatic endothelial cells (arrows; ×82) H: Pelvic MRI after treatment with sirolimus (1 mg/day for 2 years; T2-weighted imaging). Note the marked shrinkage of the pelvic lymphangioleiomyomas, a normalized sigmoid colon, and the disappearance of the interior fluid.

and interior fluid collection (Fig. 2B). Upon lymphatic scintigraphy, the accumulation of the injected label was present in an area corresponding to that in the MRI as well as staining of the left subclavian area were observed at 5 minutes after subcutaneous injection of radio-labeled HSA, indicating normal lymphatic flow in the axial lymphatics. However, the marked accumulation of radioactivity in the central area of the abdomen, suggesting lymphatic leakage into the intes- 

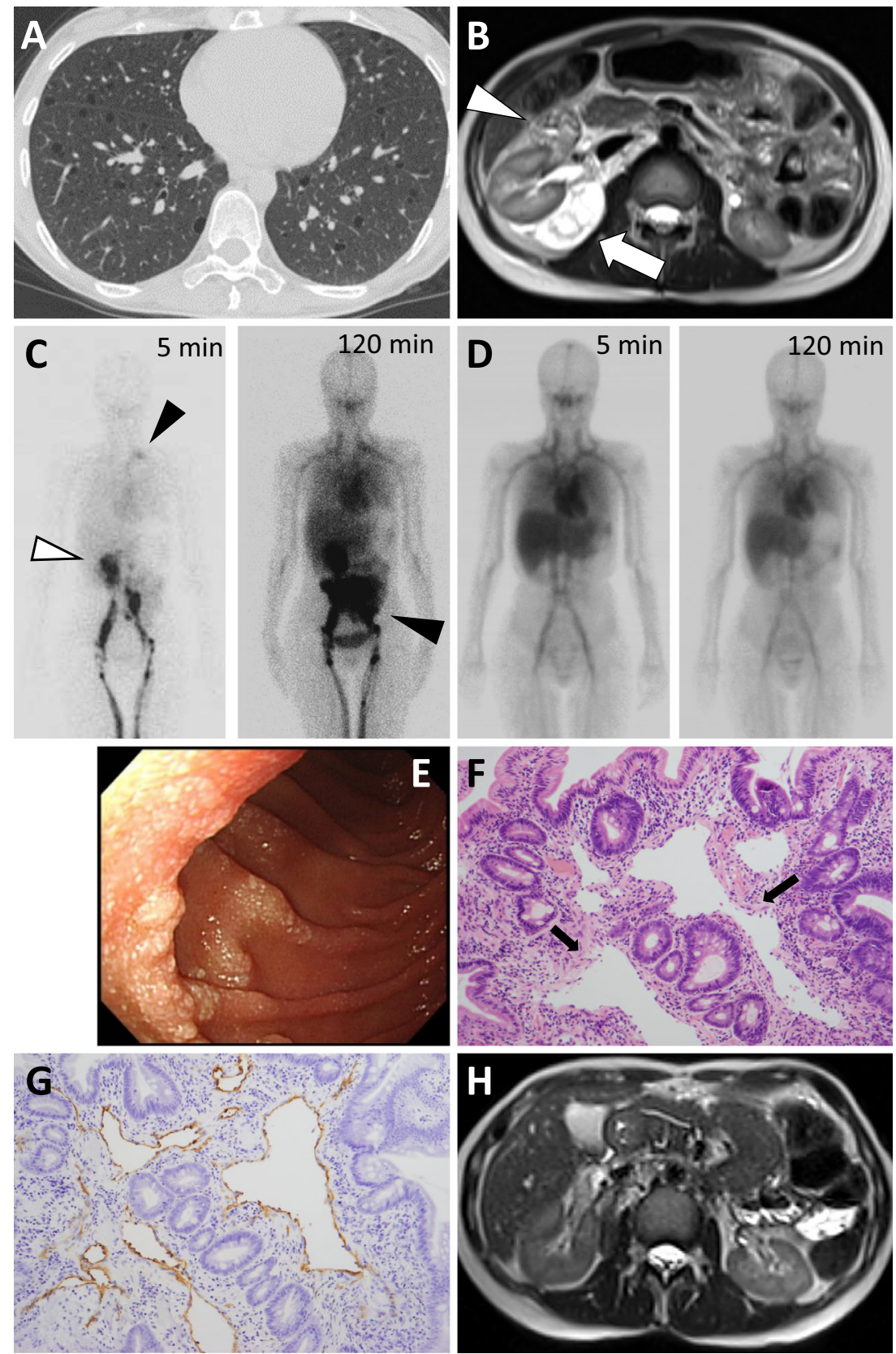

Figure 2. The clinical features of Patient 2. A: Chest CT of the multiple thin-walled cysts in both lung fields. B: Abdominal MRI (T2-weighted imaging) of multiple-lobulated cystic lesions dorsal to the right kidney (arrow), a high signal intensity was observed in the tissues around the inferior vena cava, and duodenal wall thickening with interior fluid collection can be observed (arrowhead). $\mathrm{C}$ : Lymphatic scintigraphy at 5 minutes after the subcutaneous injection of radio-labeled HSA, which accumulated in an area corresponding to that in the MR image (B) (white arrow), as well as staining of the left subclavian area (black arrow), indicating the normal lymphatic flow of the axial lymphatics. However, radioactivity was prominent in the central area of the abdomen 120 minutes later, suggesting lymphatic leakage into the intestines (black arrow). D: Protein-losing scintigraphy (intravenous injection of ${ }^{99}$ mc-labeled HSA) shows no leakage into the intestine at either 5 minutes or 120 minutes after the injection. E: Fiberoptic gastroendoscopy of the multiple whitish granules on the mucosal wall of the second portion of the duodenum. F: The biopsied duodenum with dilated, irregularly shaped thin-walled spaces (arrows) in the lamina propria of the duodenal mucosa (Hematoxylin and Eosin staining; $\times 50$ ) G: Anti-podoplanin antibody (clone D2-40) staining confirmed that the dilated, irregularly-shaped, thin-walled spaces were lymphatic vessels lined with lymphatic endothelial cells. ( $\times 50) \mathrm{H}$ : Abdominal MRI after treatment with sirolimus $(2 \mathrm{mg} / \mathrm{day}$ for 6 months) revealed that the lymphangioleiomyomas were markedly smaller, and that the duodenal wall thickening and interior fluid had disappeared. 
tines, was observed 120 minutes later (Fig. 2C). Proteinlosing scintigraphy (intravenous injection of ${ }^{99 \mathrm{~m}} \mathrm{Tc}$-labeled HSA) showed no leakage into the intestine at either 5 minutes or 120 minutes after the injection (Fig. 2D). Fiberoptic gastroendoscopy revealed multiple whitish granules on the mucosal wall of the second portion of the duodenum (Fig. 2E). The pathological examination of the biopsied duodenum revealed dilated, irregularly shaped, and thin-walled lymphatic vessels in the lamina propria (Fig. 2F, G). Based on these results, we diagnosed the patient with PLE secondary to lymphangiectasia associated with LAM.

GnRH therapy (leuprorelin acetate at $1.88 \mathrm{mg}$ every four weeks) was initiated when Patient 2 was 32 years of age, resulting in a transient improvement (TP $6.0 \mathrm{~g} / \mathrm{dL}$ and Alb 3.8 $\mathrm{g} / \mathrm{dL})$. However, this improvement did not last, and GnRH therapy was abandoned after one year. A monthly infusion of immunoglobulin was administered for one year to prevent infection, but its trough level never increased. Sulfamethoxazole/trimethoprim (800 mg/160 mg, every other day) was also initiated to prevent pneumocystis pneumonia. Accordingly, she was managed with a fat-restricted diet and the occasional use of diuretics until she participated in a clinical trial of sirolimus in Japan. At that point, her pulmonary function test revealed a $\mathrm{FEV}_{1}$ of $99.2 \%$ of the predicted value and an $\mathrm{FEV}_{1} / \mathrm{FVC}$ value of $85.8 \%$. Her laboratory data were as follows: TP, $4.4 \mathrm{~g} / \mathrm{dL}$; Alb, $2.4 \mathrm{~g} / \mathrm{dL}$; IgG, $193 \mathrm{mg} /$ $\mathrm{dL}$; and lymphocyte, $178 / \mu \mathrm{L}$. The next step was treatment with sirolimus ( $2 \mathrm{mg}$ /day; the trough level at 3 months was $5.3 \mathrm{ng} / \mathrm{mL}$ ). Its effect was remarkable. Her laboratory data at 5 months were as follows: TP, $7.1 \mathrm{~g} / \mathrm{dL} ; \mathrm{Alb}, 4.5 \mathrm{~g} / \mathrm{dL} ; \mathrm{IgG}$, $907 \mathrm{mg} / \mathrm{dL}$; and lymphocyte, 396/ $\mu \mathrm{L}$. At this point, her fatrestricted diet and the administration of sulfamethoxazole/ trimethoprim were discontinued. After 6 months of sirolimus use, abdominal MRI showed a marked reduction in the size of her lymphangioleiomyomas, and the disappearance of the duodenal wall thickening and interior fluid collection (Fig. 2H). These beneficial effects of sirolimus (2 mg/day) have continued over approximately 3 years of treatment. Sirolimus was well tolerated and only oral mucositis (grade 1), which spontaneously resolved within several months, was noted.

\section{Discussion}

We reported that two patients with LAM complicated by PLE were successfully treated with sirolimus in addition to conventional therapy. PLE appears to be very rare among LAM patients; to our knowledge, only two such cases had been reported. Kinoshita et al. described a 29-year-old woman with LAM and associated PLE (3). In that case, abdominal CT and lymphoscintigraphy revealed enlarged cystic lymphangioleiomyomas. Fiberoptic gastroendoscopy showed multiple whitish granules on the mucosal wall of the second portion of the duodenum, and a mucosal biopsy confirmed intestinal lymphangiectasia. Lin et al. published a similar case study of a 29 -year-old woman (4) in whom ab- dominal CT indicated large abdominal lymphangioleiomyomas, while a duodenal mucosal biopsy revealed intestinal lymphangiectasia.

The typical endoscopic findings of intestinal lymphangiectasia include white plaques and either white-tipped or non-white villi. Ohmiya et al. noted that the white villi type (white plaques and white-tipped villi) as reflecting the pathological feature of dilated lymphatic vessels in the lamina propria, while the non-white villi type represented the dilation of the lymphatic vessels in the submucosal layer or deeper (5). In our study, Patient 2 had similar white villi, confirming the dilation of the lymphatic vessels in the lamina propria. There are few case reports of lymphangiectasia of the colon (6-8). According to these reports, the endoscopic findings include an edematous mucosa, polyposis-like lesions and giant folds, likely duplicating the edematous mucosal changes that we reported in Patient 1. Although these endoscopic findings are sufficiently suggestive of intestinal lymphangiectasia, pathological examinations should be performed to confirm the presence of dilated lymphatics in the digestive tracts of patients with $\operatorname{PLE}(9,10)$.

The mechanisms underlying the development of intestinal lymphangiectasia are not fully understood. The condition could be primary, due to congenital lymphatic malformation, or secondary, as a consequence of impaired lymphatic flow due to a mechanical obstruction of the lymphatic vessels, cardiac disease, or portal hypertension (11). In our two patients (as well as the preceding reports), local congestion via mechanical obstruction of the lymphatic route by lymphangioleiomyomas was the most likely cause of intestinal or colonic lymphangiectasia. Both CT and MRI showed large lymphangioleiomyomas in the pelvic retroperitoneal area (Patient 1) or around the upper pole of the right kidney (Patient 2). These lesions seemed large enough to compress the lymphatic flow from the intestine or colon to the main lymphatic trunk via the mesentery. After the initiation of sirolimus treatment, the marked shrinkage of these lymphangioleiomyomas was confirmed by CT or MRI; accordingly, PLE decreased. This result strongly suggests that the reduced size of the lymphangioleiomyomas normalized the lymphatic flow from the digestive tract to the main lymphatic trunk. It is also possible that the direct invasion and destruction of the lymphatic routes by LAM cells was involved. However, no LAM cells were detected in the duodenal or colonic biopsy specimens of our patients; thus, the explanation that proliferating LAM cells were directly involved in blocking lymphatic routes is unlikely. Alternatively, there are reports of patients with PLE caused by portal hypertension (12). Thus, the obstruction of the portal system by lymphangioleiomyomas could be the cause of PLE. Nevertheless, in our patients, neither abdominal CT nor MRI revealed obvious the compression of the portal vein.

Interestingly, the clinical manifestations related to PLE in Patient 1 were not as severe as those in Patient 2. Patient 1 did not have hypogammaglobulinemia or leg edema. Differ- 
ences in the site of blocked mesenteric lymphatic collectors may be a reason for these clinical variations. The lymphatics in the digestive trunk flow into the thoracic duct via mesenteric lymphatic collectors. The severity of PLE depends on the location where mesenteric the lymphatic collectors are obstructed. That is, the blockage of the central mesenteric collectors is associated with more severe PLE in comparison to a peripheral blockage (13). Judging from the imaging studies, the lymphangioleiomyomas in Patient 2 at the mesenteric lymphatic collectors were situated more centrally than those (the retroperitoneal pelvic region) in Patient 1, resulting in the clinical differences that were observed these two patients.

The treatment of PLE consists of the management of the underlying disease and the maintenance of a healthy nutritional status. The mainstay of nutritional therapy is a low-fat and high-protein diet with medium-chain triglyceride supplementation (14). Some patients may require additional therapies, including octreotide, antiplasmin, corticosteroids, intravenous albumin, and immunoglobulin, or surgery if the lymphangiectasia is localized (15). On the other hand, it has been challenging to devise treatments for the underlying disease in order to ameliorate PLE. Sirolimus, however, has proven to not only stabilize the decline of pulmonary LAM (16) but to also reduce the size of chylous effusion and to shrink lymphangioleiomyomas in LAM patients (17). The reduction of lymphangioleiomyomas via the administration of sirolimus has been the most effective treatment strategy for our patients' PLE. Sirolimus recently proved effective for improving the condition of a patient with primary intestinal lymphangiectasia complicated with tuberous sclerosis complex in whom no obstructive lesion was evident (18). Using lymphatic endothelial cells (LECs) derived from the mesenteric lymphatic tissue of mice, Luo et al. reported that sirolimus inhibited the cells' proliferation, motility, and lymphatic tube formation (19), partly by downregulating their expression of VEGF receptor-3. Thus, it is possible that sirolimus corrected the lymphatic flow in our patients not only by reducing the size of their lymphangioleiomyomas but also by regulating the function of the LECs.

There is currently no consensus regarding when sirolimus treatment should be initiated for LAM patients. Formerly, in situations where LAM was complicated by PLE, Kinoshita et al. used low-fat diet therapy alone, which alleviated the patients' hypoalbuminemia and leg edema (3). Accordingly, we think that sirolimus should be initiated once a fatrestricted diet proves to be ineffective. Based on its longterm effectiveness, sirolimus should be a candidate for further study.

The authors state that they have no Conflict of Interest (COI).

\section{References}

1. Contini P, Schiavina M, Schiavina R, et al. Efficacy of hormonal suppression in a patient with chyluria due to lymphangioleiomyomatosis. Multidiscip Respir Med 6: 313-317, 2011.

2. Iwamoto I, Fujino T, Douchi T. Chylous vaginal discharge in a patient with lymphangioleiomyomatosis. Am J Obstet Gynecol 199: 5-6, 2008.

3. Kinoshita A, Odagi I, Aoki Y, et al. A case of lymphangioleiomyomatosis associated with protein-losing gastroenteropathy. Nihon Shokakibyo Gakkai Zasshi 105: 1775-1780, 2008 (in Japanese).

4. Lin CC, Lee TC, Liu KL, Lin JT, Lin MT, Wang HP. Education and imaging. Gastrointestinal: lymphangioleiomyomatosis with protein-losing enteropathy. J Gastroenterol Hepatol 21: 1860, 2006.

5. Ohmiya N, Nakamura M, Yamamura T, et al. Classification of intestinal lymphangiectasia with protein-losing enteropathy: white villi type and non-white villi type. Digestion 90: 155-166, 2014.

6. Asakura H, Tsuchiya M, Katoh S, Kobayashi K, Yonei Y, Yoshida T. Pathological findings of lymphangiectasia of the large intestine in a patient with protein-losing enteropathy. Gastroenterology 91: 719-724, 1986.

7. Iwao T, Yamashita F, Kuboyama S. Lymphangiectasia of the colon: report of a case. Gastroenterol Endosc 29: 328-332, 1987 (In Japaese, Abstract in English).

8. Nishitani R, Suzuki Y, Kobayashi K, Matuhashi O, Matuoka A, Osaku T. A case report, of colonic lymphangiectasia. Nihon Shokakibyo Gakkai Zasshi 6: 330-334, 1973 (in Japanese, Abstract in English).

9. Waldmann TA, Steinfeld JL, Dutcher TF, Davidson JD, Gordon RS Jr. The role of the gastrointestinal system in "idiopathic hypoproteinemia". Gastroenterology 41: 197-207, 1961.

10. Freeman HJ. Small intestinal mucosal biopsy for investigation of diarrhea and malabsorption in adults. Gastrointest Endosc Clin N Am 10: 739-753, 2000.

11. Freeman FJ, Nimmo M. Intestinal lymphangiectasia in adults. World Journal of Gastrointestinal Oncology 3: 19-23, 2011.

12. Stanley AJ, Gilmour HM, Ghosh S. Transjugular intrahepatic portosystemic shunt as a treatment for protein-losing enteropathy caused by portal hypertension. Gastroenterology 111: 1679-1682, 1996.

13. Fox U, Lucani G. Disorders of the intestinal mesenteric lymphatic system. Lymphology 26: 61-66, 1993.

14. Jeffries GH, Chapman A, Sleisenger MH. Low-fat diet in intestinal lymphangiectasia - Its effect on albumin metabolism. N Engl J Med 270: 761-766, 1964.

15. Chih PC, Yee C, Chung PL, et al. Surgical resection of duodenal lymphangiectasia: a case report. World J Gastroenterol 9: 28802882, 2003.

16. McCormack FX, Inoue Y, Moss J, Singer LG, Strange C, Nakata $\mathrm{K}$. Efficacy and safety of sirolimus in lymphangioleiomyomatosis. N Engl J Med 364: 1595-1606, 2011.

17. Angelo M, Taveira D, Hathaway O, Stylianou M, Moss J. Changes in lung function and chylous effusions in patients with lymphangioleiomyomatosis treated with sirolimus. Ann Intern Med 154: 797, 2011.

18. Sarah FP, Alexandra LG, Elizabeth AT, Uzma S. Primary intestinal lymphangiectasia treated with rapamycin in a child with tuberous sclerosis complex (TSC). Am J Med Genet 167: 2209-2012, 2015.

19. Luo Y, Liu L, Rogers D. Rapamycin inhibits lymphatic endothelial cell tube formation by downregulating vascular endothelial growth factor receptor 3 protein expression. Neoplasia 14: 228-237, 2012.

The Internal Medicine is an Open Access article distributed under the Creative Commons Attribution-NonCommercial-NoDerivatives 4.0 International License. To view the details of this license, please visit (https://creativecommons.org/licenses/ by-nc-nd/4.0/).

\footnotetext{
(C) 2017 The Japanese Society of Internal Medicine
} http://www.naika.or.jp/imonline/index.html 